\title{
L'Orphée Refoulé
}

\section{Eunice Dutra Galéry}

«On ne lit bien que ce qu' on lit dans un certain dessein tout personnel».

(PAUL VALERY)

L'approche d'un texte littéraire est toujours l'approche du mystère. A la différence qu'au mystère devrait correspondre un dévoilement, une solution, tandis que le texte littéraire ouvre des voies multiples, chaque lecture étant un nouveau dévoilement et proposant un nouveau mystère, de façon telle que le texte n'est jamais complètement dévoilé, il renaît de lui même comme le phoenix de la légende.

Mais le lecteur est en quelque sorte libre de choisir sa voie, de suivre les pistes qu'il a découvertes, pourvu qu'il soit comme le jouer de whist décrit par Poe, qui

"faz em silêncio, um grande número de observações e inferências. Seus companheiros talvez façam outro tanto, e a diferença quanto à extensão da informação assim obtida não reside tanto na validez da inferência quanto na qualidade da observação. O que é necessário é saber o que observar."1

1. POE, Edgar Allan. Os crimes da rua Morge. in: Histórias extraor. dinárias. Trad. de Breno da Silveira e outros. São Paulo, Abril Cultural, 1978. p. 109-150. 
Il y a des auteurs dont l'approche est plus facile, d'autres, comme Valéry, des maîtres à "nier ce qui est et d'expliquer ce qui n'est pas", 2 dont l'abordage pose toujours des problèmes: auteur trop hermétique pour quelques-uns, trop clair pour quelques autres, souvent on oublie la part de l'ironie dans l'oeuvre du poète - et l'ironie n'est qu'un "masquage", une sorte de procédé par dénégation de ce qui est, pour le rendre plus évident. C'est ce procédé par dénégation la maille que nous avons prise pour procéder à une lecture d'Eupalinos.

Nous nous proposons de montrer comment, par une sorte de glissement sémantique, Valéry passe d'un sujet à l'autre, ou mieux, comment Eupalinos est constitué de deux textes, l'apparent et le caché, comme une musique en contre-point, où un thème suit l'autre, plus subtil mais aussi important et toujours présent. Notre méthode sera celle préconisée par Derrida, selon laquelle

"Il faudrait (...) d'un seul geste, mais dédoublé, lire et écrire. Mais celui-là n'aurait rien compris au jeu qui se sentirait du coup autorisé à en rajouter, c'est-à-dire à ajouter n'importe quoi. Il n'ajouterait rien, la couture ne tiendrait pas. Réciproquement ne lirait même pas celui que la "prudence méthologique", les "normes de l'objectivité" et les "garde-fous du savoir" retiendraient d'y mettre du sien." 3

S'il est bien vrai que Valéry est un écrivain hanté par l'obsession de la forme, il n'est pas moins vrai que ses écrits "spontannés" constituent, pour paraphraser Valéry lui-même, un "amas" d'idées et de reflexions, où les sujets semblent ne pas avoir aucun rapport les uns avec les autres. Ce n'est pas en vain que le poète les nomme Mélange, Tel Quel ou Variété, titres qui indiquent bien que le contenu des oeuvres n'est pas le résultat d'un travail ordonné et méthodique.

2. ROUSSEAU, J.J. La Nouvelle Héloise, cité par Poe, op. cit. p. 150 (em francês no original).

3. DHRRIDA, Jacques. La Pharmacie de Platon. Tel Quel, Paris, 1968. nọ 32 , p. 4 . 
Pourtant, entre la perfection formelle des vers et les pensées notées pêle-mêle au lever du jour, il y a de ressemblances: des images qui reviennent, les mêmes dichotomies, des préoccupations esthétiques

Un troisième type d'ècrits, les écrits de "circonstance" prém sentent aussi des similitudes avec les deux autres. Ainsi Eupalinos ou l'Architecte, écrit à la demande de la revue "Architectures", "qui fixa même le nombre de lettres (155.800) que devait avoir (son) essai.", 4 en est un exemple.

Apparemment Valéry y parle de plusieurs sujets: l'architecture, la construction des bateaux, les figures géométriques, la musique, l'objet trouvé, etc., sujets qui n'ont aucun rapport apparent avec la poésie.

Notre lecture se propose de montrer ces rapports, à travers l'étude de quelques points qui ont attiré notre attention, c'est-adire, le système d'images/métaphores opposées deux à deux, qui se trouvent comme une constante dans l'oeuvre de Valéry; les "pistes" que l'écrivain nous laisse dans le texte d'Eupalinos, comme un défi ironique au lecteur, et, finalement, les dénégations à propos de plusieurs aspects du texte. Pour justifier l'inclusion de ces dernières, nous nous appuyons sur la définition freudienne de la dénégation, qui nous dit que

"El enjuiciamiento es el sustitutivo intelectual de la represión,

y su "no" un signo distintivo de la misma, un certificado de origen, algo asi como el MADE IN GERMANY." 5

Nous ne nous réclamons pas de la découverte du sens du texte valéryen; nous nous proposons une nouvelle lecture de celui-ci, tâche assez difficile, car

"un texte n'est pas un texte que s'il cache au premier regard, au premier venu, la loi de sa composition et la règle de son jeu". 6 v. 3 .

4. VALĒRY, Paul, Oelvves complètes. Paris, Pléiade, 1960. p. 1399-400,

5. FREUD, Sigmund. Obras Completas Madrid, Biblioteca Nueva, 1973. p. 2884. v. 3 .

6. DERRIDA, J. op. cit. p. 3 
Plus que d'une herméneutique, nous proposons une démarche heuristique pour la lecture de ce texte dont la double trame n'est certes pas perçue au premier regard.

Il faut d'abord, dans Eupalinos, remarquer la situation singulière des personnages, puisqu'il ne s'agit pas des êtres vivants, Socrate et Phèdre poursuivent un dialogue au-delà de la mort. Cette situation n'est pas sans relation avec les préoccupations de Valéry, puisque les morts sont "des purs esprits" et une dichotomie toujours présente dans son oeuvre est celle du pur/impur, non pas dans le sens moral, mais dans le sens chimique du terme: un corps pur, celui qui est composé d'une seule substance sans mélange.

Or, pour Valéry, l'homme, composé d'intellect et de chair, est un mélange qui empêche la connaissance totale. Les hommes sont faits d'alternances de connaissance intellectuelle et de sensations qu'ils n'arrivent pas à compreendre, ils ont "un corps qui leur permet de sortir de la connaissance et d'y rentrer." 7

Ce même étonnement et cette même idée de pur/impur se trouvent, par exemple, dans Mon Faust, dans le dialogue entre Lust et Méphistophélès:

“- Mon coeur vous soit abscur!... Il me l'est à moi-même. $(\ldots \ldots \ldots \ldots \ldots \ldots \ldots)$ Oh! mon coeur, tu te moques du mal. . . et même du bien... Mais vous!. . Tu n'es qu'esprit!... Mais les Anges eux-mêmes, les Archanges fidèles, tous ces fils de lumière et ces puissances de ferveur ne peuvent pas comprendre...

Ils sont purs, ils sont durs, ils sont forts." 8

Cette dichotomie se présente dans bien d'autres passages de Valéry, presque comme une pensée obsessionnelle. Valéry a voulu éloigner le sens moral des termes, mais lui-même joue sur la polysémie de ces mots, et dans Mon Faust, Méphistophélès emploie le mot pur dans le sens de pureté morale:

7. VALRRY, Paul, op. cit, p. 79.

8. Id. Ibidem., op. cit. v. 2, p. 349. 
"- Voyez, très pure enfant, comme je suis discret" 9

et le double emploi remet en cause les mots de Lust: les anges seraient-ils purs dans quel sens? Nous nous retrouvons devant

"ce pharmakon, cette "médecine", ce philtre, à la fois remède et poison (qui) s'introduit déjà dans le corps du discours avec toute son ambivalence." 10

Dans Eupalinos Socrate déclare que les morts "sont trop simplifiés $(\ldots)$ pour ne pas subir jusqu'au bout le mouvement de quelque idée." 11 Nous pourrions espérer par là qu'une idée serait développée justqu'à sa fin. Rien de pareil. Eupalinos se dessine comme un vrai brassage d'idées, sans fil apparent pour les lier.

Em parlant d'Eupalinos, Phèdre parle exactement comme Valéry lui-même parle à propos de la poésie. La méthode de construction d'Eupalinos ressemble étrangement à l'écriture de Valéry:

“- Il (Eupalinos) prédisait leur avenir monumental aux informes amas de pierres et de poutres qui gisaient autour de nous; et ces matériaux, à sa voix, semblaient voués à la place unique où les destins favorables à la déesse les auraient assignés. Quelle merveille que ses discours aux ouvriers! Il n'y demeurait nulle trace de ses difficiles méditations de la nuit." 12

Nous nous retrouvons ici devant la dichotomie chaos (amas informes)/ordre (la place unique), ordre résultant d'un mouvement de l'esprit aussi bien que de la parole, le chaos lié à nuit, rejeté et ordonné par l'intelligence. Le même mouvement est perçu dans la poésie de Valéry:

9. Id. ibid., v. 2, p. 345 .

10. DERRIDA, J. op. cit. p. 8 .

11. VALERY, op. cit, v. 2 p. 79.

12. Id. Ibid, op. cit. v. 2, p. 83. 
"La confusion morose

Qui me servait de sommeil

Se dissipe dès la rose

Apparence de soleil" 13

ou bien dans Rhumbs, toujours suivant ce mouvement chaos/ordre, le chaos et le sommeil liés, travail inconscient qui doit être mis en ordre par l'intelligence au réveil:

"Le sommeil a brouillé le jeu, battu les cartes; et les songes ont tout mêlé, tout remis en question...

Au réveil il y a un temps de naissance, une naissance de toutes choses avant que quelqu'un n'ait lieu..."14

plus près encore d'Eupatinos, voici un autre passage d'Autres Rhumbs, où, en plus, sont employés des termes propres à l'architecture:

"Rêve.

Eveillé, mon esprit tout à coup abandonne les choses voisines et se met à bâtir dans le monde où les constructions ne coûtent rien, ou presque rien. Une grande activité se remarque dans le demi-univers réservé aux combinaisons et tendent à me faire ce qui me plaise exactement.

Je renverse leurs projets. Je reprends; je modifie, je perfectionne." 15

La métaphore de la construction, si chère à Valéry, revient dans un autre passage d'Eupalinos; décrivant encore les procédés de l'architecte, son extrême souci du détail, la prévision des moindres ciselures dans les pierres, Phèdre reproduit les paroles mêmes de l'architecte:

13. id. ibid. v. 1, p. 3 .

14. id. ibid. 2, p. 659

15. Id. ibid. op. cit. v. 2, p. 651. 
"- Phèdre, me disait-il, plus je médite sur mon art, plus je l'exerce; plus je pense et agis, plus je souffre et me réjouis en architecte; - et plus je me ressens moi-même, avec une volupté et une clarté toujours plus certaines.

Je m'égare dans mes longues attentes; je me retrouve par les surprises que je me cause; et au moyen de ces degrés successifs de mon silence, je m'avance dans ma propre édification; et j'approche d'une si exacte correspondance entre mes voeux et mes puissances, qu'il me semble avoir fait de l'existence qui me fut donnée, une sorte d'ouvrage humain.

A force de construire, me fit-il en souriant, je crois bien que je me suis construit moi-même." 16

A cette description du travail de l'architecte, répondent plusieurs endroits de l'oeuvre critique de Valéry, aussi bien que de sa poésie. Le rapport entre la création architecturale et la création littéraire devient assez clair dans ces mots que Valéry écrit à propos de Flaubert:

"La poésie a obtenu son constructeur de temples qui taillait les mots longuement comme des pierres dures." 17

Ensuite, pour le thème de l'attente qui permet d'avancer, nous trouvons presque les mêmes mots, dans "Aurore",

"Dans mon âme je m'avance

Tout ailé de confiance" 18

et dans "Les Pas":

"Tes pas, enfants de mon silence,

Personne pure, ombre divine,

Qu'ils sont doux, tes pas retenus!

Dieux! ... tous les dons que je devine

Viennent à moi sur ces pieds nus! 19

16. id. ibid. p. $91 / 92$

17. Id. Ibid, op. cit. p. 1403 , v. 2 .

18. id. ibid. p. lll, v. 1.

19. Id. ibid. p. 120 , v. 1. 
La dernière proposition d'Eupalinos, le rapport entre le créateur et la chose créée, trouve son écho dans le passage suivant:

"L'oeuvre modifie l'auteur. $(\ldots \ldots \ldots \ldots \ldots \ldots \ldots \ldots)$

Il se reconstruit en quelque sorte en formateur

de l'ensemble réalisé, qui est un mythe.

De même un enfant finit par donner à son père

l'idée, et comme la forme et la figure de la paternité." 20

Il faut se rappeler que le passage qui suit immédiatement à celui-ci s'appelle "Créateur créé."

Cette redondance de thèmes nous paraît assez signifiante - et elle se retrouve un peu partout dans les écrits de Valéry, étant presque un système de travail:

"Je conçois, quant à moi, que le même sujet

et presque les mêmes mots pourraient être repris indéfiniment et occuper toute une vie". 21

S'il revient presque obsessivement aux mêmes images, c'est qu' elles reflètent une préoccupation fondamentale du poète, qui se dédouble en critique et se conduit en scientifique, délimitant son champ d'observation, pour mieux se connaître et pour atteindre son but, selon ses propres mots:

"Ma seule constance, mon seul instinct permanent fut sans doute de me représenter de plus en plus nettement mon fonctionnement mental et de garder ou de reprendre, aussi souvent que possible, ma liberté contre les illusions et les parasites que nous impose l'emploi inévitable du language." 22

20. Id. ibid. p. 674, v. 2 .

21. Id. Ibid. v. 2, p. 553.

22. cité par MONDOR, H. Précocité de Valéry. Paris, Gallimard. 1957. p. 122 . 
Devant ces données nous ne sommes pas surpris de retrouver les mêmes images dans des écrits sur la poésie et dans les vers de Valéry. Ce qui nous paraît signifiant c'est de les retrouver dans des écrits tels qu'Eupalinos, où apparemment Valéry traite de sujets complèment étrangers à la poésie. Ce que nous cherchons à montrer c'est que, à travers tous les sujets traités dans Eupalinos, se poursuit, comme à contre-point, le discours sur la poésie. La manière de construire est la même, le rejet de "l'objet trouvé" dans Eupalinos:

“(...) Que cet objet singulier fût l'oeuvre de la vie, ou celle de l'art, ou bien celle du temps et un jeu de la nature, je ne pouvais le distinguer... Alors, je l' ai tout à coup rejeté à la mer." 23 (C'est moi qui souligne)

va de pair avec le rejet du "vers donné":

"A la moindre rature, - le principe de l'inspiration totale est ruiné - L'intelligence efface ce que le dieu a imprudemment créé." 24

bref, à chaque thème d'Eupalinos répond le même thème dans les écrits sur la création poétique ou même dans la poésie. Manière d'affirmer en niant: le principe même de la dénégation.

Nous pourrions continuer à décrire des procédés analogues pour chaque thème traité dans Eupalinos - ce travail exhaustif nous semble pourtant inutile, puisque les exemples donnés nous semblent assez probants.

Et quant à la dénégation dont nous avons parlé plus haut, elle se produit aussi dans un autre domaine: à plusieurs reprises Valéry nie l'influence de Platon, comme dans les notes de l'édition de la Pléiade, que nous utilisons ici:

23. VALERY, op. cit., v. 2, p. 120.

24. Id. ibid. p. 550 . 
- Vous avez pourtant subi l'influence de Platon dans vos dialogues socratiques?

- Absolument pas. J'en avais lu qualques pages. Si je savais mieux le grec, il est possible que cela m'eût intéressé. Mais il est plus simple d'inventer que de reprendre. GRAECUM EST, NON LEGITUR.

- Vous n'avez pas utilisé d'ouvrages grecs, de traductions?

- Aucunement. 25

Cette négation est retrouvée dans la correspondance avec Paul Souday:

"Et quant au grec, je suis malheureusement demeuré un écolier des plus médiocres, qui se perd dans l'original de Platon et le trouve, dans les traductions, terriblement long et souvent ennuyeux..." 26

Des traces de I'influence platonicienne se trouvent pourtant dans Eupalinos, et pour le répérer il suffit de le comparer au Phèdre de Platon:

\section{Phèdre 27}

Vois-tu là bas ce platane si élevé? ( $p .218)$

Tournons par ici et descendons

l'Ilissos; (p. 218)

Dis-moi, Socrate, n'est-ce pas ici près, au bord de I'Ilissos, que Borée enleva dit-on, Orythye? (p. 218)

\section{Eupalinos}

...ni l'ombre délicate du platane; (p. 90)

Hẻlàs! pauvre Ilissus!

Et ce temple hors les murs, auprès de l'autel de Borée, te souvient-il?

Celui d'Artémis la Chasseresse? (p. 82)

25. Id. ibid. p. 1398.

26. Id. Ibid. op. cit. p. 1401.

27. PLATON. Oeuvres completes. Paris, Garnier, 1950. v. 3. 
Non, c'est plus bas, à quelques

deux ou trois stades, là

où l'on passe l'eau pour aller

au temple d'Agra *; il y a, à

cet endroit même un autel de

Borée (p. 219)

Phèdre (ou De la Beauté;

genre moral) (p. 215)

Dans Phèdre, Platon parle du discurs amoureux et du discours politique

- Un jour nous avons été par là. Nous avons discouru de la Beauté... (p. 82)

- O Phèdre, tu n'es pas sans avoir remarqué dans les discours les plus importants, qu'il s'agisse de politique ou des intérêts particuliers des citoyens, ou encore dans les paroles délicates que l'on doit dire à un amant, lorsque les circonstances sont décisives... (p. 84)

\section{Phèdre}

- Tu es si casanier que tu n'as jamais franchi la frontière et il semble bien que tu n'es jamais sorti des murs. (p. 220

- C'est le désir de m'instruire qui en est la cause; car ni les champs ni les arbres ne veulent rien m'apprendre, mais bien les hommes qui sont dans la ville.

\section{Eupalinos}

- Une chose, Socrate, une seule t'a fait défaut. $\mathrm{Tu}$ fus homme divin, et tu n'avais peut-être nul besoin des beautés matérielles du monde. Tu n'y goutais qu'à peine. (p. 90)

- $\mathrm{Tu}$ voyais toujours autre chose.

- L'homme, et l'esprit de l'homme. (p. 91)

Nous avons deux textes assez proches: les mêmes personnagens qui dialoguent, le même endroit, des allusions évidentes au sujet traité dans Phèdre, dans Eupalinos, le caractère de Socrate, à qui Phèdre reproche de ne pas s'intéresser aux choses de la nature et dont la réponse est la même dans les deux ouvrages:

* Agra, Surnom D'Artémis 
Socrate s'intéresse à l'homme; en plus, Valéry lui-même laisse échapper cet aveu:

"je ne suis pas helléniste: je n'ai jamais lu de Platon que les débuts de ses dialogues, qui m'ont toujours semblé merveilleux. Dans mes dialogues, je me suis servi de vieux souvenirs de versions grecques." 28

(c'est moi qui souligne)

La présence de Platon se fait encore voir dans la lettre que Valéry écrit à Gide, en 1891:

"Sur ma table voici d'abord Le Banquet de Platon, (.....) Quelques cahiers de notes concernant l'ornement et l'architecture. Quelques feuillets de figures géométriques. Mais cela n'est rien: écrasant, dominant, $(\ldots \ldots \ldots \ldots \ldots)$ mes bons livres de marine sont là $(\ldots \ldots \ldots \ldots){ }_{29}$

Valéry est pris au piège du "cráteur créé: après avoir déclaré qu'il "est plus simple d'inventer que de reprendre" il se trouve empêché de reconnaître l'influence de Platon dans ses dialogues. Le procéclé est si subtil qu'il le mène jusqu'à nier le sous-texte caché. Si le sous-titre - rappelé par Valéry — du Phèdre est "de la Beauté", en réalité Platon y parle surtout à propos du discours. Mais Valéry ne peut pas le reconnaître, ce serait nier l'originalité de son oeuvre. Il appelle son Eupalinos "oeuvre de circonstance", il y met, comme sur sa table de 1891, des propos sur l'architecture, sur les figures géométriques, sur les bateaux. De cette "table" publiée en 1924, le seul et grand absent c'est justement Platon.

Le système de dénégation affirme chaque fois davantage le discours en contre-point d'Eupalinos: la réflexion sur la création artistique et spécialement sur la création littéraire. Ce n'est pas en vain que tout le texte valéryen se rattache à la parole: en parlant d'Eupatinos, Phèdre déclare:

28. VALERY, op, cit. p. 1397/8, v. 2.

29. Id. Ibid, op. cit. p. $1398 / 9$, v. 2 . 
"- Je lui trouvais la puissance d'Orphée." 30

et Orphée est, ainsi que le géomètre valéryen, celui qui construit à travers la parole, puisque "véritablement, la parole peut construire" 31

Valéry ne le savait que trop, lui qui, dans le Paradoxe sur l'architecte

"évoque, en un bois thessalien, Orphée, sous les myrtes; $(\ldots \ldots \ldots \ldots)$. Le dieu chante, et, selon le rythme toutpuissant, s'élèvent au soleil les fabuleuses pierres, et l'on voit grandir vers l'azur incandescent, les murs d'or harmonieux d'un sanctuaire." 32

Encore une affirmation du véritable thème d'Eupalinos se trouve à la dernière réplique de Socrate:

“il n'y a pas d'ici, et tout ce que nous venons de dire est aussi bien un jeu naturel du silence de ces enfers, que la fantaisie de quelque rhéteur de l'autre monde qui nous a pris pour marionnettes! 33 (c'est moi qui souligne)

Cette réplique nous dévoile encore un peu plus la signification $\mathrm{du}$ texte. Les marionnettes sont des poupées mues par quelqu'un qui reste invisible du public, de vrais personnages dans le sens étymologique du mot: personae, masques sous lesquels se cache l'auteur, le "rhéteur de l'autre monde", celui qui écrit sur le discours, sur le langage poétique et sur la création littéraire, en tenant, tout comme Phèdre "le texte ou, si l'on vent, le PHARMAKON, caché sous le manteau". ${ }^{34}$

30. Id. ibid. p. 83 .

31. Id. Ibid, op. cit. v. 2, p. 111 .

32. Id. ibid. p. 1405.

33. Id. ibid. p. 147.

34. DERRIDA, op. cit. p. 


\section{BIBLIOGRAPHIE}

BARTHES, Roland. O grau zero da escritura. Trad, de Anne Arnichard e Älvaro Lorencini. São Paulo, Cultrix, 1971.

BELLEMIN-NOEL, Jean et alii. Les critiques de notre temps et Valéry. Pari,s Garnier, 1971.

BEMOL, Maurice. La méthode oritique de Paul Valéry. Paris, Nizet, 1960. CELEYRETTE-PIERRI, Nicole. Valèry et le moi. Paris, Klincksiek, 1979. DERRIDA, Jacques. La pharmacie de Platon. Tel Quel, Paris, no 32, 1968. F'REUD, Sigmund. Obras Completas. Madrid, Biblioteca Nueva, 1973. $3 \mathrm{v}$. JULIEN-CAIN, L. Trois essais sur Panl Valéry. Paris, Gallimard, 1958.

LAWLER, James R. Lecture de Valéry. Paris, PUF, 1963.

MONDOR, Henri. Précocité de Valéry. Paris, Gallimard, 1957.

NOULET, Emilie. Paul Valêry. Paris, Grasset, 1938.

POE, Edgar Allan. Histórics extraordincirias. Trad. de Bueno da Silveira e outros. São Paulo, Abril Cultural, 1978.

PLATON. Oeuvres complètes. 'Trad. d'Emile Chambry. Paris, Garnier, 1950. 3 v.

ROBINSON, Judith. L'architecture ouverte de «La Jeune Parque» Poétique, Paris, n. 37, 1979.

VALîRY, Paul. Oeuvres complètes. Paris, Pléiade, 1960, 2 v.

Charmes; commentés par Alain. Paris, Gallimard, 1952. 